\title{
DNA hypermethylation within TERT promoter upregulates TERT expression in cancer
}

\begin{abstract}
Donghyun D. Lee, ${ }^{1,2,3}$ Ricardo Leão, ${ }^{2,4}$ Martin Komosa, ${ }^{1,2}$ Marco Gallo, ${ }^{5}$ Cindy H. Zhang, ${ }^{1,2}$ Tatiana Lipman, ${ }^{1,2}$ Marc Remke, ${ }^{6}$ Abolfazl Heidari, ${ }^{1,2}$ Nuno Miguel Nunes, ${ }^{1,2}$ Joana D. Apolónio, ${ }^{7,8}$ Aryeh J. Price, ${ }^{2}$ Ramon Andrade De Mello, ${ }^{7}$ João S. Dias, ${ }^{8}$ David Huntsman, ${ }^{9}$ Thomas Hermanns, ${ }^{10}$ Peter J. Wild, ${ }^{11}$ Robert Vanner, ${ }^{2}$ Gelareh Zadeh, ${ }^{12}$ Jason Karamchandani, ${ }^{13}$ Sunit Das, ${ }^{2}$ Michael D. Taylor, ${ }^{2}$ Cynthia E. Hawkins, ${ }^{2}$ Jonathan D. Wasserman, ${ }^{14}$ Arnaldo Figueiredo, ${ }^{4}$ Robert J. Hamilton, ${ }^{15}$ Mark D. Minden, ${ }^{16}$ Khalida Wani, ${ }^{17}$ Bill Diplas, ${ }^{18}$ Hai Yan, ${ }^{18}$ Kenneth Aldape, ${ }^{16}$ Mohammad R. Akbari, ${ }^{3,19}$ Arnavaz Danesh, ${ }^{16}$ Trevor J. Pugh, ${ }^{16}$ Peter B. Dirks, ${ }^{2}$ Pedro Castelo-Branco, ${ }^{7}$ and Uri Tabori ${ }^{1,2,20}$

1Program in Genetics and Genome Biology, and ${ }^{2}$ The Arthur and Sonia Labatt Brain Tumour Research Centre, The Hospital for Sick Children, Toronto, Ontario, Canada. ${ }^{3}$ Institute of Medical Science, Faculty of Medicine, University of Toronto, Toronto, Ontario, Canada. ${ }^{4}$ Faculty of Medicine, University of Coimbra, Coimbra, Portugal. ${ }^{5}$ Departments of Physiology and Pharmacology, Biochemistry and Molecular Biology, University of Calgary, Calgary, Alberta, Canada. ${ }^{6}$ Department of Pediatric Oncology, Hematology, and Clinical Immunology, Medical Faculty, University Hospital Düsseldorf, Düsseldorf, Cermany. ${ }^{7}$ Department of Biomedical Sciences and Medicine, and ${ }^{8}$ Centro Hospitalar Universitário do Algarve, Faro, Portugal. ${ }^{9}$ Department of Pathology and Laboratory Medicine, University of British Columbia, Vancouver, British Columbia, Canada. ${ }^{10}$ Department of Urology, University Hospital Zürich, and ${ }^{11}$ Institute of Pathology and Molecular Pathology, University Hospital Zürich, University of Zürich, Zürich, Switzerland. ${ }^{12}$ Division of Neurosurgery, University of Toronto, Toronto Western Hospital, University Health Network, Toronto, Ontario, Canada. ${ }^{13}$ Department of Pathology, Montreal Neurological Institute, McGill University, Montreal, Quebec, Canada. ${ }^{14}$ Division of Endocrinology, The Hospital for Sick Children, Toronto, Ontario, Canada. ${ }^{15}$ Division of Urology, Department of Surgery, University of Toronto, Toronto, Ontario, Canada. ${ }^{16}$ Princess Margaret Cancer Centre, University Health Network, Toronto, Ontario, Canada. ${ }^{17}$ Department of Pathology, University of Texas MD Anderson Cancer Center, Houston, Texas, USA. ${ }^{18}$ Department of Pathology, Duke University Medical Center, Durham, North Carolina, USA. ${ }^{19}$ Women's College Research Institute, University of Toronto, Toronto, Ontario, Canada. ${ }^{20}$ Division of Hematology/ Oncology, The Hospital for Sick Children, Toronto, Ontario, Canada.
\end{abstract}

Replicative immortality is a hallmark of cancer cells governed by telomere maintenance. Approximately $90 \%$ of human cancers maintain their telomeres by activating telomerase, driven by the transcriptional upregulation of telomerase reverse transcriptase (TERT). Although TERT promoter mutations (TPMs) are a major cancer-associated genetic mechanism of TERT upregulation, many cancers exhibit TERT upregulation without TPMs. In this study, we describe the TERT hypermethylated oncological region (THOR), a 433-bp genomic region encompassing $52 \mathrm{CpG}$ sites located immediately upstream of the TERT core promoter, as a cancer-associated epigenetic mechanism of TERT upregulation. Unmethylated THOR repressed TERT promoter activity regardless of TPM status, and hypermethylation of THOR counteracted this repressive function. THOR methylation analysis in 1,352 human tumors revealed frequent (>45\%) cancer-associated DNA hypermethylation in 9 of 11 (82\%) tumor types screened. Additionally, THOR hypermethylation, either independently or along with TPMs, accounted for how approximately $\mathbf{9 0 \%}$ of human cancers can aberrantly activate telomerase. Thus, we propose that THOR hypermethylation is a prevalent telomerase-activating mechanism in cancer that can act independently of or in conjunction with TPMs, further supporting the utility of THOR hypermethylation as a prognostic biomarker.

\section{Introduction}

Replicative immortality is an attribute of cancer cells governed by telomere maintenance (1). Telomeres are repetitive nucleoprotein structures that protect chromosomal ends and shorten after each replicative cycle, playing important roles in genome stability and cancer prevention $(2,3)$. To achieve replicative immortality, approximately $90 \%$ of human cancers reactivate telomerase - a holoenzyme responsible for elongating telomeres - through reexpression of the catalytic subunit telomerase reverse transcriptase (TERT) (4).

Previous studies have identified 2 prevalent cancer-associated TERT promoter mutations (TPMs) causing a cytidine-to-thymidine

Conflict of interest: The authors have declared that no conflict of interest exists. License: Copyright 2019, American Society for Clinical Investigation.

Submitted: March 27, 2018; Accepted: October 9, 2018

Reference information: J Clin Invest. 2019;129(1):223-229.

https://doi.org/10.1172/JCl121303. change at genomic loci Chr5:1,295,228 (C228T) and 1,295,250 (C250T) as a genetic mechanism of TERT upregulation (5-9). However, TPMs do not prevent initial bulk telomere shortening at the time of malignant transformation and only act on cells with critically short telomeres to delay replicative senescence (10), implying that TPMs alone are insufficient for cancer cells to exhibit the telomerase activity required for telomere length maintenance. Moreover, common tumor types, including breast, lung, prostate, colorectal, and hematological malignancies rarely exhibit TPMs (11-16) yet display telomerase activity, supporting the idea that other undefined TERT-upregulating mechanisms must exist.

One mechanism that has not yet been thoroughly investigated is the epigenetic regulation of TERT. Previous seminal studies have reported an association between TERT promoter hypermethylation and elevated TERT expression in cancer $(17,18)$, leading to our recent work, which uncovered this association in a specific region within the TERT promoter, termed upstream of the 


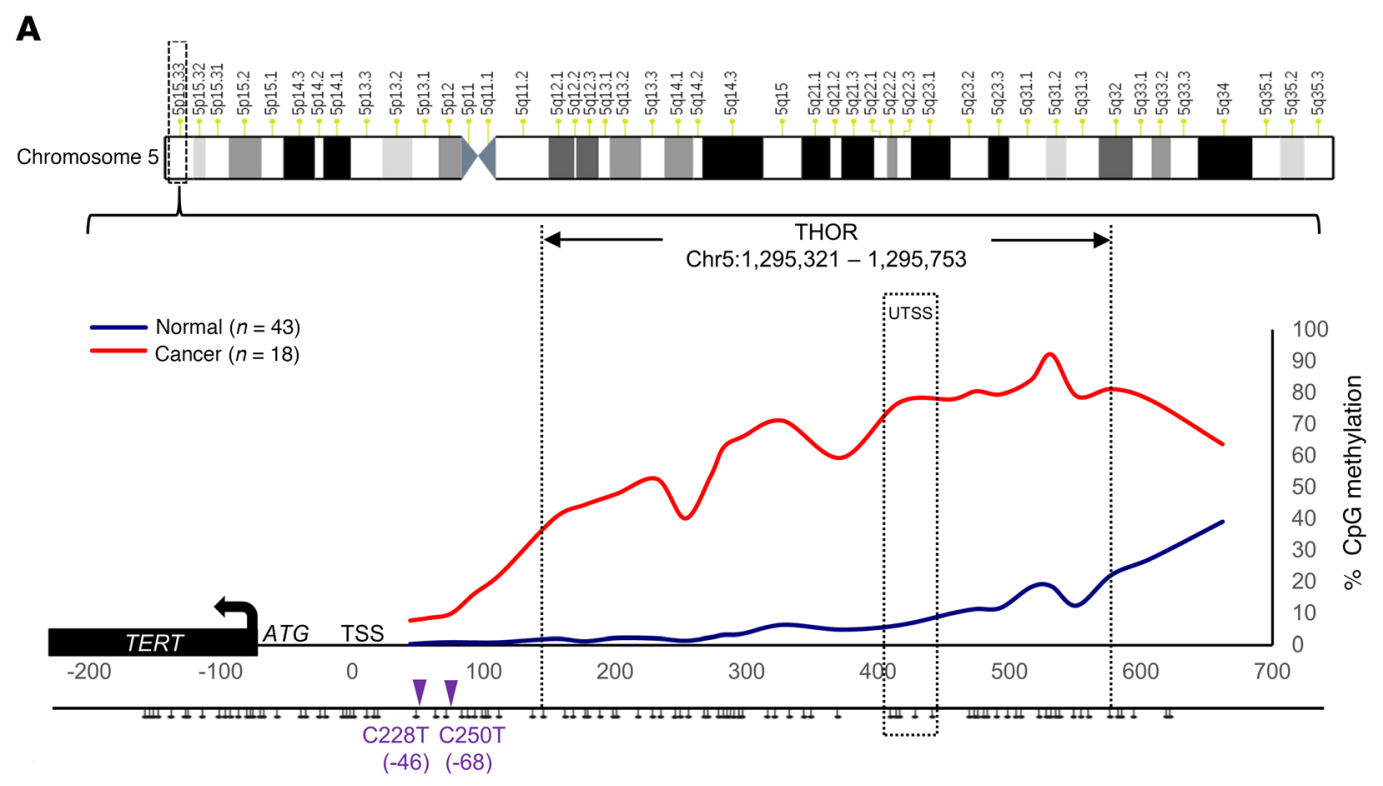

B
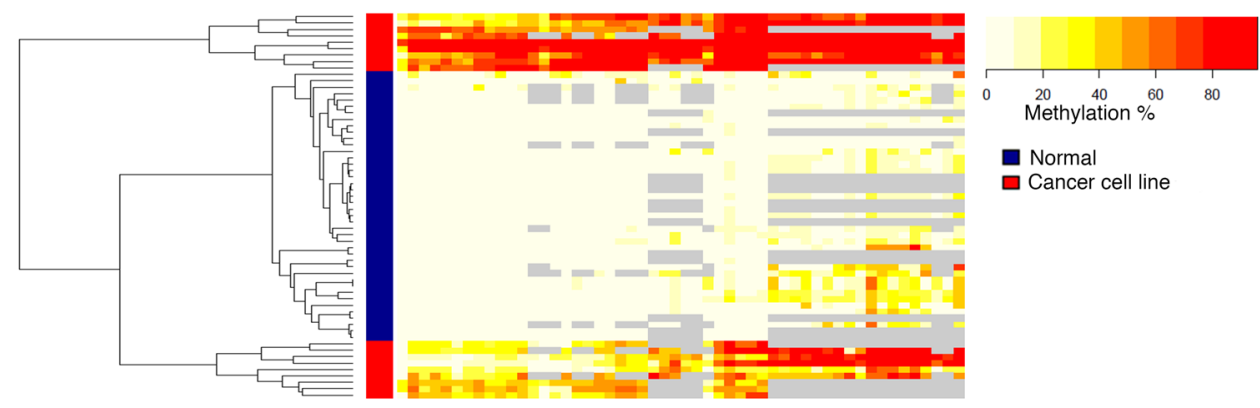

Figure 1. Defining THOR through DNA CpG methylation analysis of the TERT promoter. (A) Average CpG methylation of the TERT promoter in normal cell lines and tissues ( $n=43$, blue) and TERT-expressing cancer cell lines ( $n=18$, red). THOR is a 433-bp region ( -140 to -572 , relative to the TSS) comprising 52 CpG sites and located adjacently upstream of the common C228T and C250T TPMs (purple triangles). The UTSS encompasses 5 CpC sites within THOR. ATC and TSS are the start codon and TSS of the TERT promoter, respectively. Lollipops represent individual CpG sites. (B) Methylation heatmap generated from unsupervised clustering displays the methylation percentage of each $\mathrm{CpC}$ site within THOR for normal cell lines and tissues ( $n=43$, blue) and $T E R T$-expressing cancer cell lines ( $n=18$, red). Gray color indicates unavailability of data.

transcription start site (UTSS) (19). Several studies have reported frequent TERT promoter (UTSS) hypermethylation in various TERT-expressing cancer types (19-23), suggesting an epigenetic mechanism of telomerase activation in multiple cancers. However, the boundaries and functional impact of this region on TERT promoter activity have not been examined in detail.

In this study, we used conventional and next-generation sequencing (NGS) to uncover TERT hypermethylated oncological region (THOR). Unmethylated THOR acts as a repressive element on TERT promoter activity, while methylation of THOR counteracts this repressive effect. Importantly, activating effects of TPMs on the TERT promoter are reduced when unmethylated THOR is present, implying that TPMs and THOR are 2 distinct TERT regulatory mechanisms. Finally, we found that THOR hypermethylation is a prevalent phenomenon in TERT-expressing tumor types, regardless of their TPM status.

\section{Results and Discussion}

We examined the methylation landscape of the TERT promoter, which included approximately $650 \mathrm{bp}$ upstream of the transcrip- tion start site (TSS), with bisulfite-sequencing of DNA samples from normal cell lines and tissues $(n=43)$ and TERT-expressing cancer cell lines $(n=18)$. Using both pyrosequencing and targeted NGS technologies, which produced highly consistent results (Supplemental Figure 1, A and B; supplemental material available online with this article; https://doi.org/10.1172/JCI121303DS1), we defined THOR as a 433-bp genomic region within the TERT promoter (Chr5:1,295,321-1,295,753, GRCh37/hg19) that encompasses $52 \mathrm{CpG}$ sites and is located upstream of common TPM sites (Figure 1A). Methylation of the CpG sites within THOR was significantly increased in cancer cell lines compared with normal samples, with a mean methylation difference of $58 \%$ across THOR $\left(P=1.49 \times 10^{-9}\right.$; Figure $1 \mathrm{~A}$ and Supplemental Figure 2). Unsupervised clustering based on the $\mathrm{CPG}$ methylation status within THOR clearly separated every TERT-expressing cancer cell line from normal samples (Figure 1B).

Increased THOR methylation across all $52 \mathrm{CpG}$ sites was validated and confirmed in tumors from various tissues $(n=87$; Figure $2 \mathrm{~A}$ ), and unsupervised clustering separated $82 \%$ (71/87) of these tumors from normal samples (Figure 2B). To examine the 
A
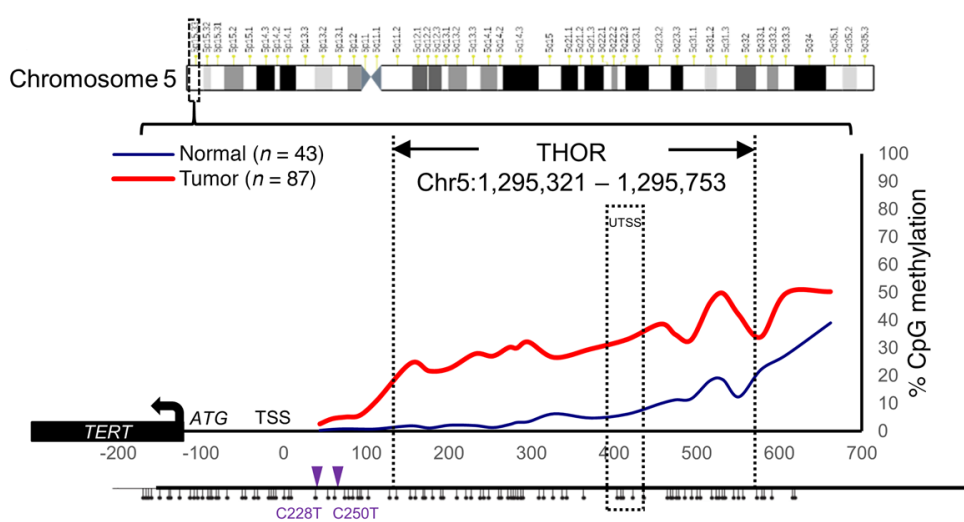

B

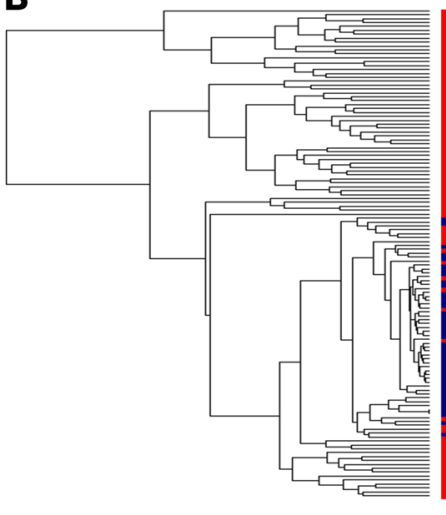

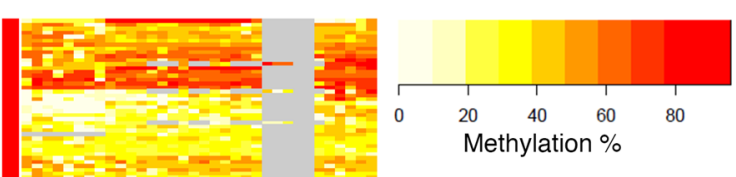

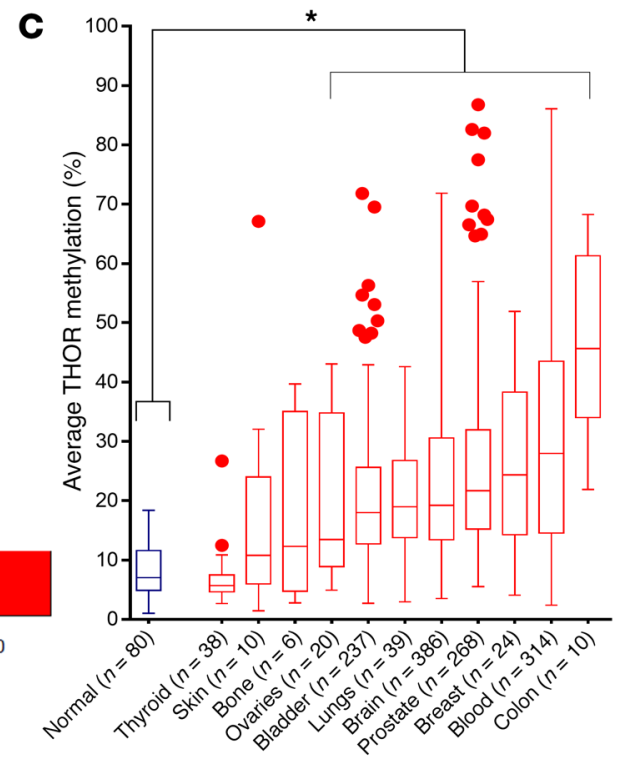

D

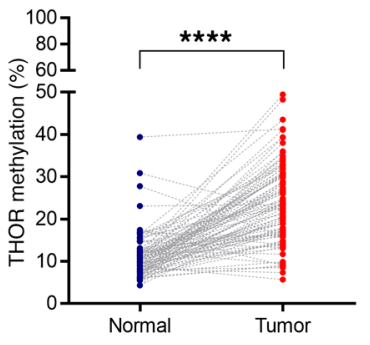

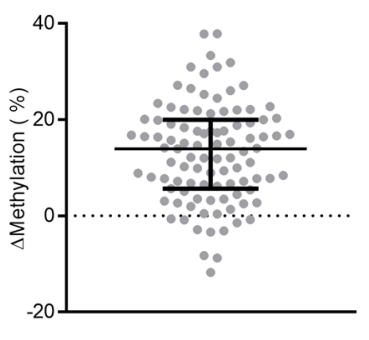

- Normal

$\square$ Tumor

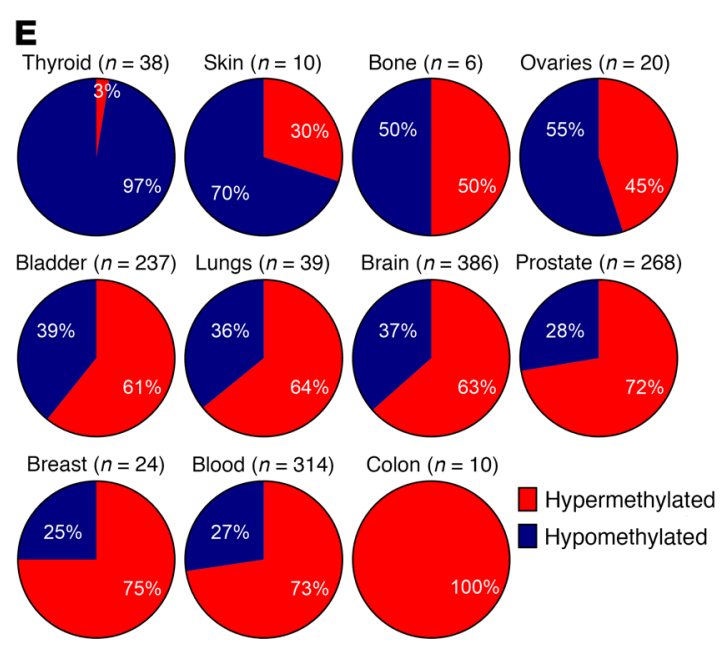

Figure 2. THOR hypermethylation is prevalent in human cancers. (A) Average DNA CpG methylation of the TERT promoter in normal cell lines and tissues ( $n=43$, blue) and tumor samples ( $n=87$, red). (B) Methylation heatmap generated from unsupervised clustering shows the methylation percentage of each CpG site within THOR for normal cell lines and tissues $(n=43)$ and tumor samples $(n=87)$. Gray color indicates unavailability of data. (C) Box-and-whisker plot shows the median and distribution of the average THOR methylation levels in normal control samples $(n=80$, blue) and samples from various tumor tissue types $\left(n=1,352\right.$; red). ${ }^{*} P<0.05$, by Sidak's multiple comparisons test. (D) Difference in average THOR methylation levels between each pair of normal tumor samples ( $n=99$; left plot) and distribution of differences in THOR methylation (right plot; median and IQR). ${ }^{* * *} P<0.0001$, by paired $t$ test. (E) Pie charts display the frequencies of the THOR hypermethylation signature across various tumor types.

extent of THOR methylation in larger tumor cohorts, we tested and confirmed that $5 \mathrm{CpG}$ sites (UTSS) (19) within THOR accurately represented the average THOR methylation (Supplemental Figure 3). Using these $\mathrm{CpG}$ sites, we screened 1,352 tumor and 80 normal samples from various tissues to assess THOR methylation in cancer (Figure 2C). The median THOR methylation of normal samples was $7.0 \%$, with no samples exceeding $18.3 \%$, while most adult cancer types exhibited significantly higher median THOR methylation $(P<0.05)$, with $91.4 \%$ of all tumors exceeding the median THOR methylation level of normal tissues. Of note, the low THOR methylation levels detected in thyroid cancers may be associated with their known lower malignant potential and better prognosis compared with other tumor types. Other tumor types such as skin and bone showing low and heterogeneous methyla- tion levels utilize other mechanisms for telomere maintenance, such as TPMs and the alternative lengthening of telomeres (ALT) pathway, respectively (24). Furthermore, we compared matched normal control and tumor samples in a subset of lung $(n=32)$ and prostate $(n=67)$ cancers, in which $89.9 \%(89 / 99)$ of the tumors had higher THOR methylation levels than matched normal tissue samples by a median of $13.9 \%$ (Figure 2D), corroborating THOR methylation as a cancer-associated epigenetic event.

We assessed the prevalence of THOR methylation in cancer by dichotomizing tumor samples as hypomethylated or hypermethylated using a cutoff value of $16.1 \%$, adding 2 SDs to the mean methylation of normal samples as previously described (see Methods) $(19,20)$. All cancer types screened had a high prevalence $(>45 \%)$ of THOR hypermethylation, with the exception of thyroid (3\%) 

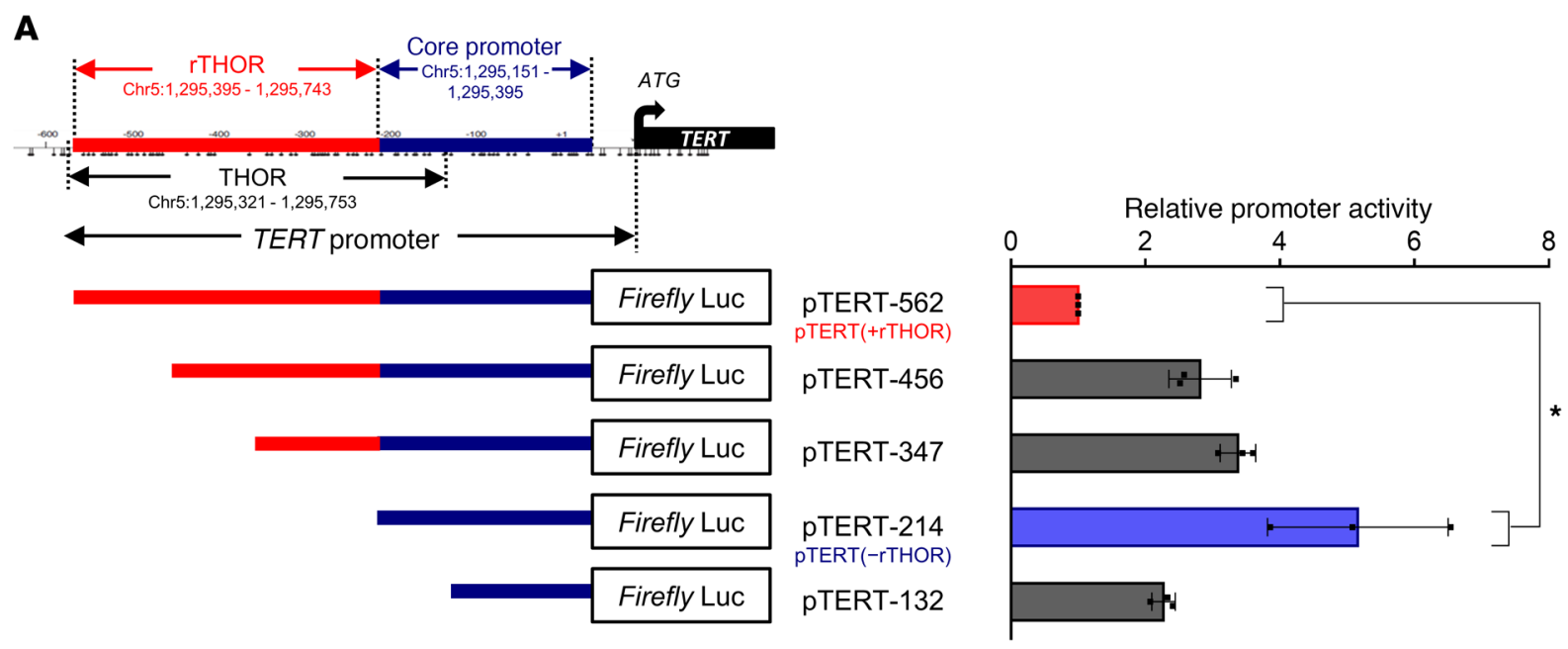

B
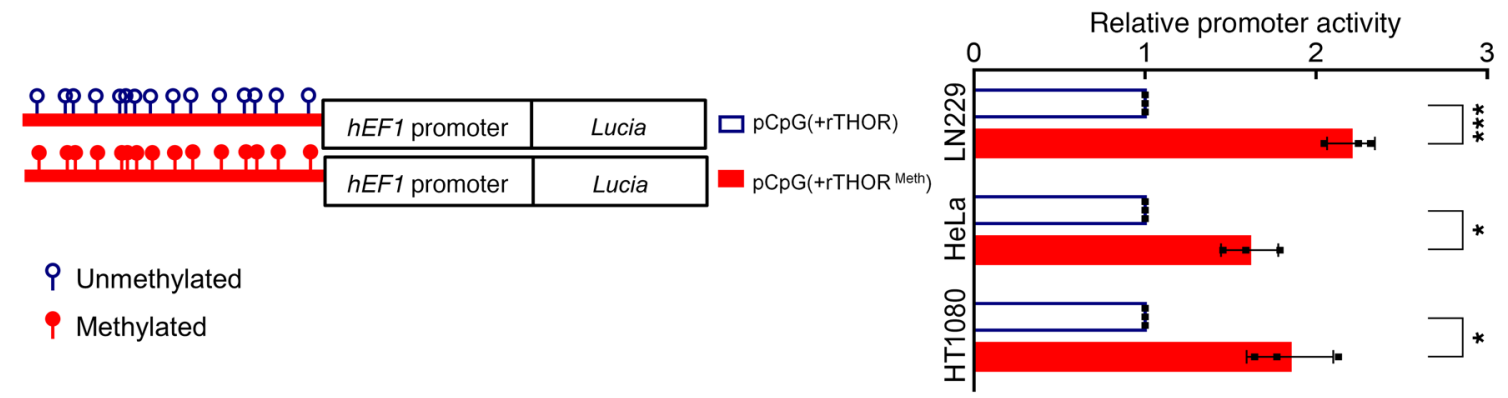

Figure 3. Hypermethylation counteracts the repressive effect of rTHOR on TERT promoter activity. For the data shown, each experiment was performed in triplicate. (A) Schematic representation of the TERT promoter. rTHOR (red) is a transcriptional regulatory element within THOR, located upstream of the TERT core promoter (blue). Normalized fold changes in TERT promoter activity are shown for the specified luciferase constructs transfected into the glioblastoma cell line LN229. The numbers in the plasmid constructs indicate the distance (bp) from the TERT TSS. ${ }^{*} P<0.05$, by unpaired $t$ test. (B) Normalized fold changes in $h E F 1$ promoter activity are shown for CpG-free constructs when rTHOR was unmethylated or methylated (in vitro) in the cancer cell lines LN229, HeLa, and HT1080. ${ }^{*} P<0.05$ and ${ }^{* *} P<0.001$, by unpaired $t$ test.

and skin $(30 \%)$ cancers (Figure 2E). Interestingly, tumors in which TPMs are rarely observed (i.e., prostate, breast, blood, and colon cancers) $(11,12,15,16)$ displayed a higher prevalence of THOR hypermethylation compared with those in which TPMs are commonly found (i.e., skin, bladder, and brain cancers) $(5,8,25)$ (Figure $2 \mathrm{E}$ ), suggesting that THOR hypermethylation may be a crucial TERT-activating mechanism in tumors without TPMs. Together, these observations highlight a potential cancer-associated mechanism of telomerase activation through THOR hypermethylation and suggest the usefulness of the THOR hypermethylation signature in differentiating normal and cancerous tissue.

To examine the role of THOR as a transcriptional regulatory element of TERT, we first performed 5 ' truncation analysis of the $T E R T$ promoter using reporter gene constructs spanning both the TPM sites and THOR. We observed a continual increase in reporter gene expression in a cancer cell line (LN229) as THOR was gradually truncated from the $5^{\prime}$ end, with the pTERT- 214 construct displaying peak promoter strength (Figure 3A). Further truncation of 82-bp (pTERT-132) resulted in an approximately 2-fold reduction of reporter gene expression, indicating that the minimal TERT core promoter is a region located up to 214 bp upstream of the TERT TSS, encompassing the TPM sites and the proximal $75 \mathrm{bp}$ of THOR. Importantly, addition of repressive THOR (rTHOR) to the
TERT core promoter resulted in a significant decrease in reporter gene expression compared with expression of the TERT core promoter alone in LN229 cells (Figure 3A) and in multiple cancer cell lines from different tissues (Supplemental Figure 4). This region, which we defined as rTHOR (Chr5:1,295,395-1,295,743), is a functional region within THOR that represses TERT expression when unmethylated. We then tested the effect of rTHOR remethylation using a unique reporter gene plasmid completely devoid of CpG sites (pCpGfree-promoter-Lucia; InvivoGen). rTHOR was cloned upstream of the modified CpG-free $h E F 1$ promoter [pCpG $(+\mathrm{rTHOR})]$ and was efficiently methylated in vitro [pCpG $\left(+\mathrm{rTHOR}^{\mathrm{Meth}}\right]$ (Supplemental Figure 5). Hypermethylation of rTHOR resulted in a significant increase in reporter gene expression in LN229 cells and in 2 additional cancer cell lines (HeLa and HT1080) (Figure 3B), implying that methylation of THOR counteracts the repressive effect of rTHOR. Finally, we treated brain cancer and glioma stem cell lines, which harbor THOR hypermethylation and high TERT expression, with the demethylation agent decitabine, resulting in reduced THOR methylation and TERT expression (Supplemental Figure 6). In contrast, we found that TERT expression was not altered in normal embryonic stem cells lacking THOR hypermethylation (Supplemental Figure 6) after treatment with decitabine. These observations infer that can- 
A
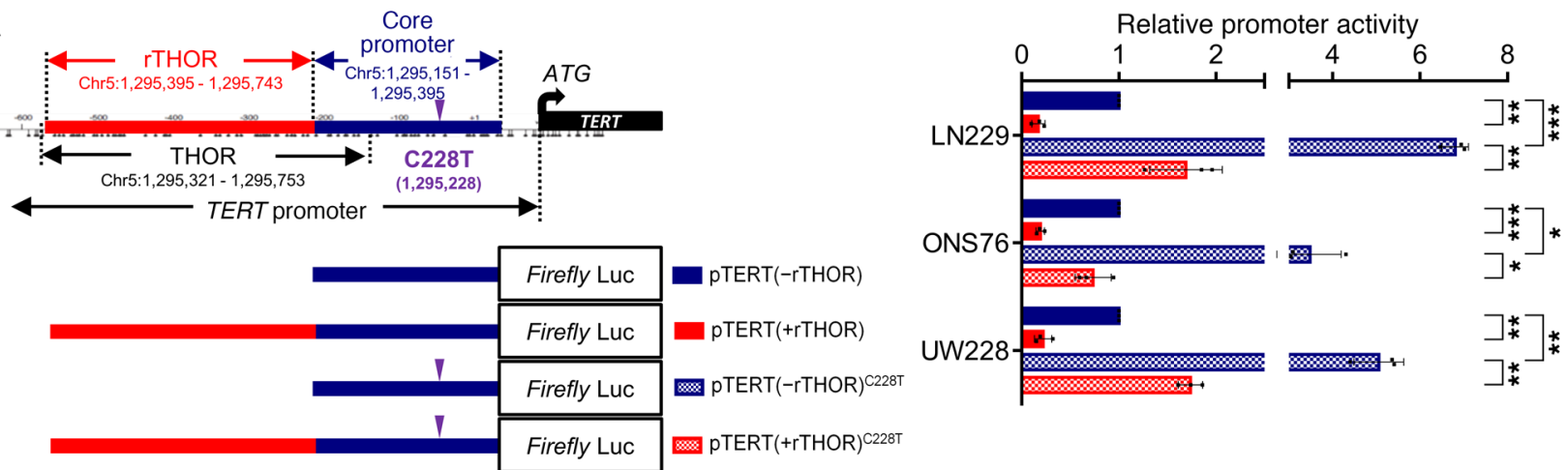

B

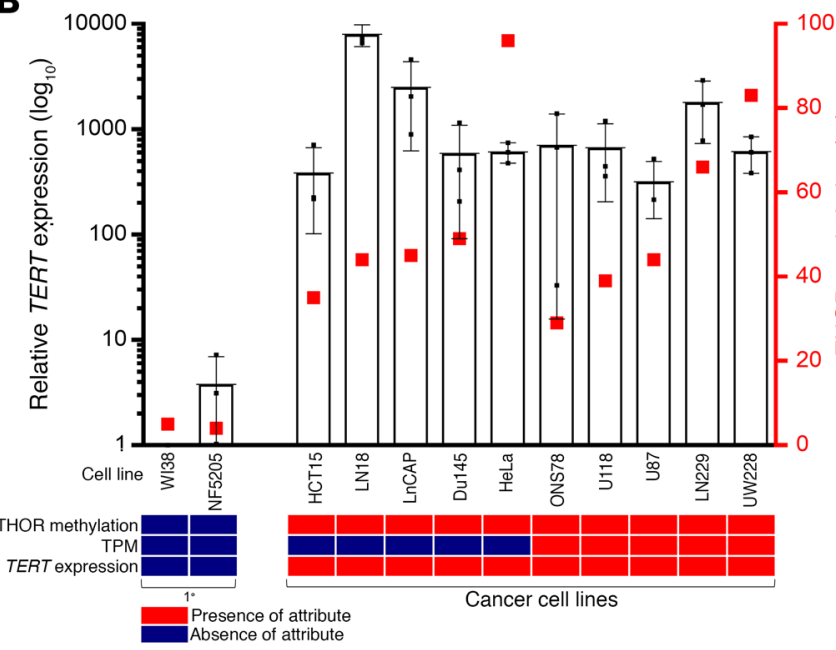

C
Tumors with low TPM frequency

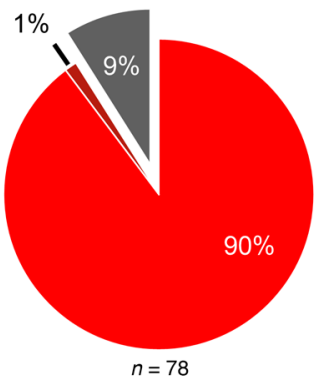

Tumors with high TPM frequency

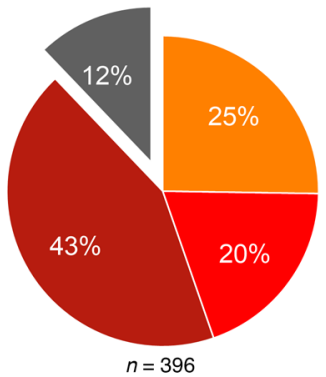

D
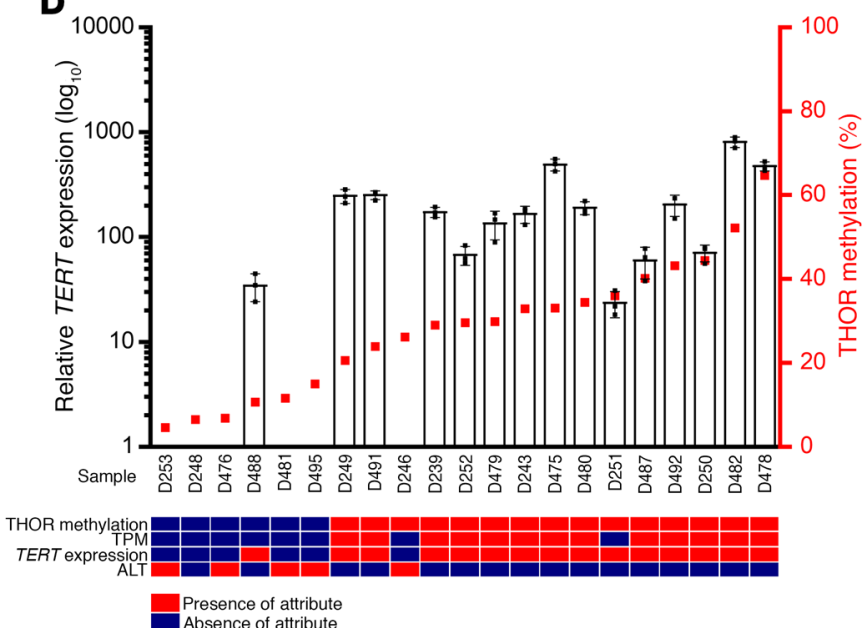

Presence of attribute

Figure 4. Coexistence and interplay of TPM and THOR hypermethylation in human cancers. For the data shown, each experiment was performed in triplicate. (A) Normalized fold changes in TERT promoter activity are shown for the specified luciferase constructs, with the presence and absence of THOR and/or C228T TPM, in the glioblastoma cell line LN229 and the medulloblastoma cell lines ONS76 and UW228. ${ }^{*} P<0.05$, ${ }^{*} P<0.01$, and ${ }^{* *} P<0.001$, by unpaired $t$ test. (B) TERT expression (mean $\pm \mathrm{SD}$, black bars and dots, $y$ axis) and average THOR methylation levels (red dots, $y$ axis) are shown in human primary $\left(1^{\circ}\right)$ and cancer cell lines. TERT regulation-associated characteristics for all cell lines are shown below the graph. (c) Pie charts display the frequencies of TPMs and the THOR hypermethylation signature in TPM-common tumors (gliomas and melanomas) and TPM-independent tumors (prostate, lung, colon, and breast). (D) TERT expression (mean \pm SD, black bars and dots, $y$ axis) and THOR methylation levels (red dots, $y$ axis) are shown in a subset of adult gliomas $(n=21)$. TERT regulation-associated characteristics for these samples are shown below the graph. cer cells hypermethylate THOR to counteract the repressive effect of rTHOR and promote TERT upregulation.

A high prevalence of THOR hypermethylation in tumor types that commonly harbor TPMs (Figure 2E) and the previous finding that TPMs alone are insufficient at preventing initial bulk telomere shortening at the time of malignant transformation (10) led us to investigate the relationship between THOR and TPMs. In contrast to the cancer-associated hypermethylation observed within THOR, the average DNA methylation of the CpG sites at the TPM locus (Chr5:1,295,225-1,295,263, GRCh37/hg19) was ubiquitously low $(<10 \%)$ in normal tissue (0.8\%), tumor samples $(4.4 \%)$, and TERT-expressing cancer cell lines (9.2\%) (Figure 1A and Figure $2 \mathrm{~A})$. This suggests that the genomic loci encompassing either
THOR or TPMs are distinct TERT regulatory regions. To investigate the functional repressive effect of unmethylated rTHOR in the context of TPMs, we compared reporter gene expression in the constructs pTERT(+rTHOR) and pTERT(-rTHOR), which contained the TERT core promoter with and without rTHOR, respectively, and in the presence and absence of C228T TPMs (Figure 4A). As expected, the addition of rTHOR to the TERT core promoter decreased promoter strength by an average of approximately 5 -fold in all 3 cancer cell lines tested, while the C228T mutation resulted in an average increase of approximately 5 -fold (Figure 4A). The addition of rTHOR to the C228T-mutated TERT core promoter effectively counteracted the activating effect of this mutation, and the activity was comparable to that of the WT TERT 
promoter without rTHOR. These findings indicate that THOR and TPMs are nonredundant but discrete regulatory mechanisms of TERT expression in cancer.

To explore the impact of these mechanisms on telomerase activation in cancer, we first examined whether they could coexist in 10 TERT-expressing cancer cell lines. All cell lines showed THOR hypermethylation and, importantly, 5 of the cell lines showed coexistence of the 2 TERT-activating mechanisms (Figure $4 \mathrm{~B})$. However, we observed no significant difference in the levels of TERT expression between the cancer cell lines when TPMs were present or absent (Supplemental Figure 7). This suggests that in certain cancers in which TPM is absent, other mechanisms such as THOR hypermethylation contribute to TERT expression. Second, we assessed the prevalence and coexistence of these mechanisms in cancer types in which TPMs are either frequently or rarely present (Figure 4C). In a cohort of cancers that commonly have TPMs (gliomas and melanomas, $n=396$ ), we frequently (43\%) observed TPMs and THOR hypermethylation together, while $45 \%$ of the tumors exhibited only 1 of the mechanisms. Meanwhile, in a cohort of cancers that lack TPMs (prostate, lung, breast, and colon cancers, $n=78$ ), 90\% of the tumors exhibited only THOR hypermethylation. Nonetheless, in both groups, the prevalence of THOR methylation, together with TPMs, reflected the overall frequency of telomerasedependent tumors ( $90 \%)$. Third, we screened a subset of gliomas, which are tumors that exhibit heterogeneous telomere maintenance mechanisms including THOR hypermethylation, TPMs, and ALT ( $n=21$ ) (Figure 4D). Interestingly, 93.3\% (14 of 15) of the TERT-expressing gliomas had THOR hypermethylation alone or with TPMs, while $83.3 \%$ (5 of 6 ) of the gliomas that lacked TERT expression were missing either one of the TERT-activating mechanisms but were primarily dependent on ALT for telomere maintenance. These observations highlight the role of THOR hypermethylation as one of the TERTupregulating mechanisms in cancer, either independently or in conjunction with TPMs.

Although we describe THOR hypermethylation as an additional TERT-upregulating mechanism, an understanding of its biological mechanism requires further investigation. A recent study has associated allele-specific hypomethylation and active histone marks (H3K4me2/3) in cancer cell lines that harbor TPMs (26). In this case, THOR hypermethylation may act as a regulatory mechanism strictly through transcription factor binding, enabling TERT expression even in the allele without active histone marks. Cis-acting transcriptional repressors such as WT1 and MZF-2 are known to bind the genomic region within THOR (27), but whether their binding is methylation sensitive requires further investigation. Another interesting aspect of THOR hypermethylation is that it may regulate other genes in the proximity to TERT that ultimately affect TERT expression. One example is the $h T E R T$ antisense promoter-associated ( $h T A P A S)$ noncoding RNA, whose promoter overlaps with the TERT promoter (28). Last, to explore the coexistence and interplay between THOR hypermethylation and TPMs in more detail, single-cell-level analyses should be performed in the future.

In summary, this study defined THOR as a region of cancerassociated DNA hypermethylation that is located adjacent to the
TERT core promoter and common TPM sites. We demonstrated that unmethylated rTHOR is a repressive element of the TERT promoter and that hypermethylation counteracts this effect, suggesting that cancer cells methylate THOR to upregulate TERT expression and activate telomerase. In addition, our study proposes 2 clinically relevant implications. First, unraveling the exact mechanism of THOR hypermethylation-driven TERT expression will uncover potential therapeutic targets for cancer treatment. Second, together with TPMs, the discovery of this TERTupregulating mechanism in cancer accounts for how approximately $90 \%$ of human cancers activate telomerase. Our findings provide biological insight into why tumors with THOR hypermethylation are associated with a poorer clinical outcome $(19,20,22,23)$, further highlighting its value as a potential prognostic biomarker.

\section{Methods}

A complete description of the methods and statistical analysis is provided in the Supplemental Materials. An unsupervised clustering heatmap was created with a modified version of Methylation plotter (29). NGS data are publicly available in the NCBI's Gene Expression Omnibus (GEO) database (GEO GSE120511).

Statistics. General statistical analyses were performed using a Fisher's exact test or a $\chi^{2}$ test for categorical variables, and a 2-tailed $t$ test (GraphPad Prism, version 6.0) for continuous variables. $P$ values of less than 0.05 were considered statistically significant.

Study approval. All experiments were performed with the approval of the research ethics board of the Hospital for Sick Children (Toronto, Ontario, Canada).

\section{Author contributions}

PCB and UT were responsible for the study concept. DDL, PCB, and UT designed the experiments. DDL conducted all in vitro experiments, methylation analyses, and statistical analyses. RL collected data and conducted methylation analyses for prostate and bladder samples. MG, CHZ, and TL conducted the decitabine treatment experiments. $\mathrm{MK}$ and $\mathrm{AD}$ performed the MiSeq data analysis, which was supervised by TJP. AH and AJP analyzed the HiSeq data. NMN and JDA assisted with data interpretation. MR, RADM, JSD, DH, TH, PJW, RV, GZ, JK, SD, MDT, CEH, JDW, AF, RJH, MDM, KW, BD, HY, KA, MRA, and PBD provided patients' samples. DDL, PCB, and UT cowrote the manuscript.

\section{Acknowledgments}

This work was supported by operating grants from the Canadian Institutes of Health Research (MOP-137899); the Canadian Cancer Society Research Institute; MaRS Innovation, and a Fundação para a Ciência e a Tecnologia Research Center grant (UID/ BIM/04773/2013 CBMR 1334).

Address correspondence to: Pedro Castelo-Branco, Universidade do Algarve, Campus de Gambelas, Dep Ciências Biomédicas e Medicina, Edificio 7, piso 3, Gab. 3.18, 8005-139 Faro, Portugal. Phone: 351.289.800.100 ext. 7813; Email: pjbranco@ualg.pt. Or to: Uri Tabori, Staff Haematologist/Oncologist, Division of Haematology/Oncology, The Hospital for Sick Children, 555 University Avenue, Toronto, Ontario, Canada, M5G1X8. Phone: 416.813.7654 ext. 201503; Email: uri.tabori@sickkids.ca. 
1. Hanahan D, Weinberg RA. Hallmarks of cancer: the next generation. Cell. 2011;144(5):646-674.

2. Blackburn EH, Gall JG. A tandemly repeated sequence at the termini of the extrachromosomal ribosomal RNA genes in Tetrahymena. JMol Biol. 1978;120(1):33-53.

3. O'Sullivan RJ, Karlseder J. Telomeres: protecting chromosomes against genome instability. Nat Rev Mol Cell Biol. 2010;11(3):171-181.

4. Finkel T, Serrano M, Blasco MA. The common biology of cancer and ageing. Nature. 2007;448(7155):767-774.

5. Arita $\mathrm{H}$, et al. Upregulating mutations in the TERT promoter commonly occur in adult malignant gliomas and are strongly associated with total 1p19q loss. Acta Neuropathol. 2013;126(2):267-276.

6. Borah S, et al. Cancer. TERT promoter mutations and telomerase reactivation in urothelial cancer. Science. 2015;347(6225):1006-1010.

7. Horn $\mathrm{S}$, et al. TERT promoter mutations in familial and sporadic melanoma. Science. 2013;339(6122):959-961.

8. Huang FW, Hodis E, Xu MJ, Kryukov GV, Chin L, Garraway LA. Highly recurrent TERT promoter mutations in human melanoma. Science. 2013;339(6122):957-959.

9. Nault JC, et al. High frequency of telomerase reverse-transcriptase promoter somatic mutations in hepatocellular carcinoma and preneoplastic lesions. Nat Commun. 2013;4:2218.

10. Chiba K, et al. Mutations in the promoter of the telomerase gene TERT contribute to tumorigenesis by a two-step mechanism. Science. 2017;357(6358):1416-1420.

11. Gay-Bellile M, et al. TERT promoter status and gene copy number gains: effect on TERT expression and association with prognosis in breast cancer. Oncotarget. 2017;8(44):77540-77551.

12. Killela PJ, et al. TERT promoter mutations occur frequently in gliomas and a subset of tumors derived from cells with low rates of self-renewal. Proc Natl Acad Sci U S A. 2013;110(15):6021-6026.

13. Lam G, Xian RR, Li Y, Burns KH, Beemon KL. Lack of TERT Promoter Mutations in Human B-Cell Non-Hodgkin Lymphoma. Genes (Basel). 2016;7(11):E93.

14. Ma X, et al. Recurrent TERT promoter mutations in non-small cell lung cancers. Lung Cancer. 2014;86(3):369-373.

15. Mosrati MA, et al. Association between TERT promoter polymorphisms and acute myeloid leukemia risk and prognosis. Oncotarget. 2015;6(28):25109-25120.

16. Stoehr R, et al. Frequency of TERT Promoter Mutations in Prostate Cancer. Pathobiology. 2015;82(2):53-57.

17. Guilleret I, Yan P, Grange F, Braunschweig R, Bosman FT, Benhattar J. Hypermethylation of the human telomerase catalytic subunit (hTERT) gene correlates with telomerase activity. Int J Cancer. 2002;101(4):335-341.

18. Zinn RL, Pruitt K, Eguchi S, Baylin SB, Herman JG. hTERT is expressed in cancer cell lines despite promoter DNA methylation by preservation of unmethylated DNA and active chromatin around the transcription start site. Cancer Res. 2007;67(1):194-201.

19. Castelo-Branco P, et al. Methylation of the TERT promoter and risk stratification of childhood brain tumours: an integrative genomic and molecular study. Lancet Oncol. 2013;14(6):534-542.

20. Castelo-Branco P, et al. A cancer specific hypermethylation signature of the TERT promoter predicts biochemical relapse in prostate cancer: a retrospective cohort study. Oncotarget.
2016;7(36):57726-57736.

21. Wang N, et al. Genetic and epigenetic background and protein expression profiles in relation to telomerase activation in medullary thyroid carcinoma. Oncotarget. 2016;7(16):21332-21346.

22. Wu Y, et al. Telomerase reverse transcriptase methylation predicts lymph node metastasis and prognosis in patients with gastric cancer. Onco Targets Ther. 2016;9:279-286.

23. Zhang H, Weng X, Ye J, He L, Zhou D, Liu Y. Promoter hypermethylation of TERT is associated with hepatocellular carcinoma in the Han Chinese population. Clin Res Hepatol Gastroenterol. 2015;39(5):600-609.

24. Gaspar TB, Henriques J, Marconato L, Queiroga FL. The use of low-dose metronomic chemotherapy in dogs-insight into a modern cancer field. Vet Comp Oncol. 2018;16(1):2-11.

25. Leao R, et al. Integrated molecular signatures of TERT promoter deregulation predict disease outcomes in non-muscle invasive bladder cancer. Ann Oncol. 2017;28(suppl_5):mdx361.056.

26. Stern JL, et al. Allele-Specific DNA Methylation and Its Interplay with Repressive Histone Marks at Promoter-Mutant TERT Genes. Cell Rep. 2017;21(13):3700-3707.

27. Kyo S, Takakura M, Fujiwara T, Inoue M. Understanding and exploiting hTERT promoter regulation for diagnosis and treatment of human cancers. Cancer Sci. 2008;99(8):1528-1538.

28. Malhotra S, Freeberg MA, Winans SJ, Taylor J, Beemon KL. A Novel Long Non-Coding RNA in the hTERT Promoter Region Regulates hTERT Expression. Noncoding RNA. 2017;4(1):E1.

29. Mallona I, Díez-Villanueva A, Peinado MA. Methylation plotter: a web tool for dynamic visualization of DNA methylation data. Source Code Biol Med. 2014;9:11. 